\title{
Failure of noninvasive prediction of pulmonary hypertension in patients with idiopathic pulmonary fibrosis
}

\author{
F. Salajka1, V. Bartoš1, J. Novosad², J. Št'ásek ${ }^{3}$, J. Bis ${ }^{3}$, M. Brtko4, \\ P. Polanský1, V. Koblížek ${ }^{1}$, V. Sedlák1
}

ABSTRACT: F. Salajka, V. Bartoš, J. Novosad, J. Št'ásek, J. Bis, M. Brtko, P. Polanský, V. Koblížek, V. Sedlák.

Background. Pulmonary hypertension (PH) in patients with advanced idiopathic pulmonary fibrosis (IPF) is a complication connected with unfavorable prognosis. Great efforts have been made in attempting to establish a reliable non-invasive method which would enable detection of this complication. In this context a formula using pulmonary function parameters was published with outstanding results.

Methods. We tested the formula in 27 IPF patients who underwent a lung function examination, cardiac ultrasonography and catheterisation on the same day.

Results. Pulmonary hypertension was detected by catheterisation in 17 patients $(63 \%)$. In our group, con- trary to the published data, the aforementioned formula was neither useful for detecting patients with a high probability of $\mathrm{PH}$ nor as a means of calculating the mean pulmonary artery pressure in individual patients $(p=0.502$ and $p=0.833$, respectively). Ultrasound examination reached borderline correlation with the values measured by catheterisation when we compare patients with relevant results $(r=0.531, p=0.051)$. However, the examination gave no usable results in 13 patients $(48 \%)$.

Conclusion. Our data suggests that no reliable, noninvasive method is currently available for detecting and confirming PH in IPF patients. We did not confirm the usefulness of the published formula. Further carefully organised studies will be necessary to verify or refute it. Monaldi Arch Chest Dis 2011; 75: 3, 172-177.

${ }^{1}$ Department of Pneumology, University Hospital and Charles University Faculty of Medicine, Hradec Kralove,

2 Department of Clinical Immunology and Allergology, University Hospital and Charles University Faculty of Medicine, Hradec Kralove,

3 First Department of Internal Medicine, University Hospital and Charles University Faculty of Medicine, Hradec Kralove,

${ }^{4}$ Department of Cardiosurgery, University Hospital and Charles University Faculty of Medicine, Hradec Kralove, Czech Republic.

Correspondence: Dr. František Salajka, Fakultni nemocnice Hradec Kralove, Plicni klinika, Sokolska 581,50005 Hradec Kralove, Czech Republic; e-mail: salajka@fnhk.cz

\section{Introduction}

Idiopathic pulmonary fibrosis (IPF) is included among pulmonary parenchyma disorders with unknown etiology and unfavorable prognosis. The incidence and mortality of IPF have yet to be precisely estimated [1]. In a recent study, the prevalence in the overall United States population was estimated to be 42.7/100 000 and 14.0/100 000, while the incidence was estimated at 16.3/100 000 and $6.8 / 100000$, according to the used criteria [2]. A study covering the years 1981-1990 gave a maximal incidence of 1.28/100 000 and a prevalence of 12.1/100 000 for the Czech population [3].

In patients with advanced IPF, pulmonary hypertension $(\mathrm{PH})$ frequently occurs as a complication associated with deterioration of clinical status and poor prognosis $[4,5]$. The incidence of $\mathrm{PH}$ among IPF patients is unclear so far, however, the clinical data as well as post-mortem studies indicate that $\mathrm{PH}$ can be present in about half of the patients $[6,7]$. However, no reliable non-invasive method for disclosing the presence of $\mathrm{PH}$ is avail- able at present. Various radiological signs and lung function parameters are used with varying reliability in the effort to determine which patients have a high probability of $\mathrm{PH}$. In addition, various indirect indices using lung function parameters were tried to improve the sensitivity and specificity of presently available instruments such as the pulmonary hypertension index (ratio of FVC/TLCO) or composite physiological index $[8,9]$. Unfortunately, the results obtained remain to this date unfavorable.

A few years ago, a formula using standard pulmonary function parameters such as forced vital capacity (FVC), transfer factor for carbon monoxide (TLCO), and oxygen saturation $\left(\mathrm{SpO}_{2}\right)$ was published with outstanding results. The final form of this formula is $-11.9+0.272 \mathrm{x} \mathrm{SpO}_{2}+0.0659 \mathrm{x}$ $\left(100-\mathrm{SpO}_{2}\right)^{2}+3.06 \times \mathrm{FVC} \% / \mathrm{TLCO} \%$ [10]. Using this formula, the mean pulmonary artery pressure (MPAP) value could provide an estimate within a $5 \mathrm{mmHg}$ precision. In addition, using a cut-off level of 21 , the presence of PH defined as MPAP greater than $25 \mathrm{mmHg}$ should be possible 
to identify in IPF patients with high sensitivity. A study demonstrating the validation of this formula in a different group of IPF patients subsequently followed [11]. The aim of our study was to confirm the usefulness of this method in our group of IPF patients.

\section{Patients and Methods}

In a prospective study, all patients followed on an outpatient basis were examined for idiopathic pulmonary fibrosis in the Department of Pneumology, University Hospital, Charles University Faculty of Medicine in Hradec Kralove. This examination was performed in order to assess the ability of the particular patient for lung transplantation. The results of examinations required as a part of the complex pre-transplantation examination were only included into the study. Before starting any examination, written consent was obtained from all the patients. The diagnosis of IPF was stated according to the ATS/ERS consensus [1] and was based on clinical criteria, lung function tests, bronchoalveolar lavage fluid and/or lung tissue biopsy examinations. During the short hospitalisation, lung function tests, echocardiographic and right heart catheterisation examinations were performed on the same day. The lung function tests included spirometry, body plethysmography, and carbon monoxide transfer factor examinations according to the ATS/ERS recommendation (ZAN 500 BODY, nSpire Health, Inc., USA). Blood gas examinations were performed in blood samples taken from the radialis artery by a blood gas analyzer (ROCHE OMNI S1, Roche Diagnostics, Hoffmann-La Roche Ltd). Then, transthoracic cardiac ultrasonography was performed (SONOS 5500, Royal Philips Electronics Inc, The Netherlands), followed by a right heart catheterisation (Cathcor, Siemens-Elema AB, Solna, Sweden). All of the examinations were performed by specialists blinded to the results of the previous examinations. Pulmonary hypertension was defined as MPAP greater than $20 \mathrm{mmHg}$. For statistical evaluation, the 17.0 version of SPSS Statistics software was used. Spearman $\mathrm{r}$ was applied to explore mutual correlations between distinct predictors. A value of $p \leq 0.05$ was assigned as statistically significant. A receiver operator characteristics curve (ROC) was applied to analyze cut-off values of feasible predictors to estimate the presence of pulmonary hypertension. Logistic regression was applied to express odds ratios of the presence of pulmonary hypertension with respect to distinct predictors.

\section{Results}

We organised the complex examination of a total of 27 patients with IPF. The mean age of the 17 men and 10 women was $60.7 \pm 7.4$ years. The mean length of the interval between establishing the diagnosis and the examination was $5.6 \pm 3.5$ years. The mean values of the basic lung function parameters are presented in the table (table 1). In total, pulmonary hypertension (mean arteria pul- monalis blood pressure measured by catheterisation $>20 \mathrm{mmHg}$ ) was detected in 17 patients $(63 \%)$. Individual data concerning the catheterisation, values calculated using the published formula [10] and ultrasonographic examination from all 27 patients are presented in table 2 .

First, we tried to identify parameters of lung function eligible to be used as possible predictors of pulmonary hypertension. Taking the mean arteria pulmonalis blood pressure measured by catheterisation (cMPAP) as the "golden standard", no significant correlation was found with the "pulmonary hypertension index" expressed as FVC/TLCO. When evaluating various cut-offs, no significance was reached in our group for any cut-off level, including the originally used level $1.4(\mathrm{r}=-0.115, \mathrm{p}=0.569)$.

According to the published data, the recently presented formula should be able to predict pulmonary hypertension with a high sensitivity $(95 \%$ with a specificity of $58 \%$ ) when the calculated value with a cut-off greater than 21 is used [11]. For this calculation, pulmonary hypertension was defined as MPAP from catheterisation greater than $25 \mathrm{~mm} \mathrm{Hg}$. In our group, the significant correlation was not reached for the $\mathrm{PH}$ defined according to the recent recommendation (MPAP > $20 \mathrm{mmHg}$, $\mathrm{r}=0.135, \mathrm{p}=0.502)$. Also, when the border level criterion for PH was heightened to $25 \mathrm{mmHg}$, similar to the originally published data, no significant correlation was found $(r=-0.043, p=0.833)$. According to the analysis of the ROC values, both insufficient sensitivity and specificity were calculated for various cut-off levels of the formula (table 3 ). Although the exact value of 21.0 used in the original article was not obtained, the closest values and their sensitivity and specificity are pointed out. It is obvious, that no cut off value can be used according to our results to reach sufficient sensitivity.

Following the publication with the tested formula, we tried to use the estimated lung function parameters to calculate the level of MPAP in individual patients [11]. In our group, the use of the formula was not helpful in this way. In individual patients, the mean difference between MPAP measured at catheterisation and calculated by the formula was 9.6 and reached up to $44 \mathrm{mmHg}$. Taking

Table 1. - Basic parameters of lung function

\begin{tabular}{lcc}
\hline Parameter & Mean \pm SD & Median \\
\hline VCmax (\%) & $66.4 \pm 20.4$ & 63.0 \\
FVC $(\%)$ & $64.4 \pm 19.9$ & 62.0 \\
$\mathrm{TLCO}\left(\mathrm{mmol} . \mathrm{min}^{-1} \cdot \mathrm{kPa}^{-1}\right)$ & $39.3 \pm 14.3$ & 37.0 \\
$\mathrm{PaO}_{2}$ in rest $(\mathrm{kPa})$ & $9.0 \pm 2.0$ & 8.8 \\
$\mathrm{SpO}_{2}$ in rest $(\%)$ & $92.9 \pm 5.9$ & 95.0 \\
$\mathrm{cMPAP}(\mathrm{mm} \mathrm{Hg})$ & $25.9 \pm 10.6$ & 24.0 \\
\hline
\end{tabular}

VCmax: maximal vital capacity; FVC: forced vital capacity; TLCO: transfer capacity for carbon monoxide; $\mathrm{PaO}_{2}$ : arterial partial pressure of oxygen; $\mathrm{SpO}_{2}$ : oxygen blood saturation; cMPAP: mean arteria pulmonalis blood pressure measured by catheterisation. 
Table 2. - Individual data from all 27 patients

\begin{tabular}{cccccc}
\hline Sex & Age & cMPAP & fMPAP & cSPAP & uSPAP \\
\hline M & 62 & 35 & 17 & 52 & 50 \\
M & 67 & 30 & 20 & 49 & $70-75$ \\
F & 60 & 34 & 20 & 47 & less than 35 \\
M & 59 & 24 & 17 & 41 & n.a. \\
M & 56 & 20 & 24 & 32 & $30-35$ \\
M & 49 & 43 & 19 & 57 & n. a. \\
M & 55 & 32 & 64 & 62 & n. a. \\
F & 63 & 30 & 19 & 43 & $50-60$ \\
F & 67 & 18 & 22 & 31 & n. a. \\
M & 50 & 24 & 18 & 36 & $35-40$ \\
M & 62 & 30 & 20 & 48 & $40-45$ \\
M & 58 & 19 & 19 & 34 & less than 35 \\
M & 68 & 31 & 16 & 38 & n. a. \\
F & 57 & 23 & 19 & 42 & n. a. \\
F & 67 & 8 & 19 & 13 & n. a. \\
F & 58 & 28 & 21 & 39 & $30-35$ \\
F & 59 & 17 & 18 & 29 & n. a. \\
F & 69 & 14 & 21 & 28 & $60-65$ \\
F & 43 & 62 & 18 & 88 & $70-90$ \\
M & 66 & 22 & 22 & 38 & n. a. \\
M & 74 & 30 & 19 & 49 & n. a. \\
M & 66 & 22 & 17 & 39 & n. a. \\
M & 55 & 16 & 19 & 32 & n. a. \\
F & 67 & 18 & 19 & 33 & 40 \\
M & 68 & 18 & 19 & 26 & n. a. \\
F & 49 & 19 & 20 & 27 & less than 35 \\
M & 64 & 32 & 23 & 52 & $50-55$ \\
\hline
\end{tabular}

cMPAP: catheterisation mean pulmonary artery pressure; fMPAP: mean pulmonary artery pressure calculated using the tested formula [7]; cSPAP: catheterisation systolic pulmonary artery pressure; uSPAP: systolic pulmonary artery pressure estimated by ultrasonography; n. a.: not available. sults or no results at all were obtained in 17 out of 27 patients $(63 \%)$.

\section{Discussion}

In the advanced stages of idiopathic interstitial pneumonias $\mathrm{PH}$ is a frequent complication, although its exact incidence is not known. The prevalence of $\mathrm{PH}$ in patients with IPF varies greatly, anywhere from $32 \%$ to $85 \%$ of patients [13]. Also, well-documented data shows that the prognosis of patients with $\mathrm{PH}$ is significantly worse than those without PH. In a group of 79 patients with IPF, where right heart catheterisation data was available, the 1 -year mortality of patients with $\mathrm{PH}$ was $28 \%$ compared to $5.5 \%$ in those without [14]. However, despite this situation, no suitable and reliable method to reveal PH, especially in its early stadium, is yet available for routine clinical use. Pulmonary catheterisation is generally considered the gold standard for estimating the presence of $\mathrm{PH}$. However, due to the invasiveness and possible risk of serious complications (namely in less experienced centres) it cannot be regarded as the proper method for routine use in diagnostics and follow up.

Among the various indirect methods, radiological evaluation of the enlarged diameter of the right branch of the arteria pulmonalis on chest X-ray or of the main pulmonary artery on CT scan has acquired wide use. However, the reliability the interval $\pm 10 \mathrm{mmHg}$ as the sign of accuracy as was used in the ultrasound evaluation [12], the difference between the measured value and the calculated value was greater and therefore a significantly inaccurate result was obtained in 9 patients (33\%). Taking the whole group, we found only a non-significant correlation between the mean cMPAP and the calculated MPAP $(r=-0.148, p=$ $0.461)$.

Ultrasound (US) represents the most widely used non-invasive means of enabling the detection of the presence and magnitude of pulmonary hypertension. In our patients, a borderline correlation was detected between systolic PAP measured during catheterisation (cSPAP) and the values obtained by US (uSPAP) $(r=0.531, p=0.051)$. However, two weak points should be highlighted. First, this analysis was not performed on every patient, as it was not possible to get usable SPAP results in 13 patients on whom US was performed. Moreover, when comparing the individual uSPAP data in 14 patients for whom the examination brought valid results with their cSPAP and taking the interval of $\pm 10 \mathrm{mmHg}$ compared to the cSPAP as the sign of accuracy (according to 12), we found 3 patients in whom the uSPAP value was overestimated in terms of the designated tolerance, and 1 patient in whom the value was underestimated. Thus, using ultrasound, either significantly incorrect re- of this method in patients with advanced pulmonary fibrosis is questionable. While standard chest radiography may provide signs of $\mathrm{PH}$, the sensitivity of this modality is generally considered low [15]. According to some reports, the evaluation of the pulmonary artery diameter or its ratio to the aortic diameter promised more reliable results. Conversely, a study correlating the presence of $\mathrm{PH}$ proved by catheterisation with increased pulmonary artery diameter or an increased pulmonary artery/aortic diameter ratio failed to show any significant relation [18].

Similar considerations on unreliability restrict the use of echocardiography. Although this method is generally accepted as the most frequently used screening, diagnostic, and follow up method, its limitation should be carefully judged. In a recently published study, accuracy defined as a result within $\pm 10 \mathrm{mmHg}$ compared to the invasive estimation was reached in only $52 \%$ of patients with PH of various origins [19]. The magnitude of difference in this group of patients reached up to $40 \mathrm{mmHg}$. In patients with advanced lung disease the use of echocardiography to assess $\mathrm{PH}$ is even more limited. In $56 \%$ of the 374 patients (candidates for lung transplantation due to various lung diseases) it was not possible to estimate sPAP. Of those in whom ultrasound enabled pulmonary artery pressure to be estimated, the result was in- 
Table 3. - Sensitivity and specificity for particular cutoff values of the formula to predict pulmonary hypertension

\begin{tabular}{ccc}
\hline Calculated index & Sensitivity & Specificity \\
\hline 19.6300 & 0.417 & 0.667 \\
19.8750 & 0.333 & 0.667 \\
20.2300 & 0.333 & 0.733 \\
20.4900 & 0.250 & 0.733 \\
20.6050 & 0.167 & 0.733 \\
21.1350 & 0.167 & 0.800 \\
21.6350 & 0.167 & 0.867 \\
22.2350 & 0.167 & 0.933 \\
23.2000 & 0.083 & 0.933 \\
43.6950 & 0.083 & 1.000 \\
64.7800 & 0.000 & 1.000 \\
\hline
\end{tabular}

accurate (a difference greater than $10 \mathrm{mmHg}$ compared tocatheterisation) in $52 \%$ of the patients [12]. In another study which included 110 IPF patients, the sPAP could be estimated in 60 patients, with acceptable accuracy in only 24 (22\% of the whole group) [20]. A similar limitation was present in our group of patients, where in $48 \%$ ultrasound did not bring the required results and in another $15 \%$ the results were significantly incorrect compared to the catheterisation values.

Due to this situation, great effort has been made to find a non-invasive method allowing $\mathrm{PH}$ to be predicted in patients with various lung diseases, namely with IPF. Several parameters of lung function have already been used for this purpose. However, the results were largely inconclusive. Vital capacity (VC) is the most widely used parameter to express the severity of restrictive ventilatory disorders connected with IPF. However, numerous studies confirmed its unsuitability in predicting the presence of $\mathrm{PH}$, as was for instance presented in two groups of lung transplant candidates [21, 22]. Moreover, after stratifying 100 IPF patients according to FVC value into five groups, the prevalence of $\mathrm{PH}$ and level of mPAP was higher in the group with the least restriction $(\mathrm{FVC}>70 \%)$ than in the group with the worst restriction $(\mathrm{FVC}<40 \%)(55 \%$ vs $33 \%$, and 30.5 vs $21 \mathrm{mmHg}$, respectively) [23].

Another parameter closely connected to the impairment level in patients with IPF is fTLCO). Its use brought promising results in several studies. A highly significant correlation between $\mathrm{PH}$ and the TLCO level $(p<0.001)$ was found in the group of 88 patients with IPF [24]. One of the restrictions of this study is that the evidence of $\mathrm{PH}$ is provided by ultrasound only and not bycatheterisation. In two other studies which evaluated the correlation between PH occurrence proved by catheterisation and TLCO levels in patients with IPF, the same level of significance, i.e. $p=0.04$, was reached $[14,18]$. On the other hand, results of further studies questioned the validity of this parameter. No significant correlation between PH and TLCO was found in a group of 118 IPF patients [8]. A recently published study evaluating patients with an advanced form of interstitial infliction came to the same conclusion [25].
Based on experience gained in patients with systemic sclerosis, the ratio of $\mathrm{FVC} \% / \mathrm{TLCO} \%$ with a cut-off value of 1.4 was proposed as an index which would make it possible to specify the group of patients with probable $\mathrm{PH}$ [9]. A significant correlation between PH and this index (without using the recommended cut-off level) was found in one study evaluating 65 patients with IPF [18]. However, no significant correlation between MPAP and FVC/TLCO levels was found in another study [8]. The parameters of FVC and TLCO were also used to construct the composite physiological index (CPI), which was originally developed with the aim of expressing the disease severity according to the extent of the disease on the CT [26]. The CPI formula is $91.0-(0.65 \times \mathrm{TLCO} \%)$ $-(0.53 \times \mathrm{FVC} \%)+\left(0.34 \times \mathrm{FEV}_{1} \%\right)$. The usefulness of this index in predicting $\mathrm{PH}$ was also not confirmed [8].

The published formula using the $\mathrm{SpO}_{2}, \mathrm{FVC}$, and TLCO values was constructed on a retrospective review of medical records of 61 IPF patients evaluated for lung transplantation [10]. In the original group of patients, its use brought very promising results concerning the sensitivity, specificity, positive and negative predictive values. Furthermore, this formula has proved useful in clinical practice in a performed external validation involving 60 IPF patients from two different institutions [11]. In this study, the ability of the formula was proved for both estimating the individual MPAP value as well as predicting the presence of $\mathrm{PH}$. The MPAP estimated using this method was within 5 $\mathrm{mmHg}$ of thecatheterisation values in $72 \%$ of the cases. Using the calculated cut-off level $>21$ $\mathrm{mmHg}$, a sensitivity of $95 \%$ was obtained in predicting the $\mathrm{PH}$ defined as cMPAP $>25 \mathrm{mmHg}$. However, this study too was constructed on a retrospective data analysis.

In the literature available to us, we came across only one article focusing on independent validation of this formula in a population of IPF patients [27]. In this prospective study, 37 IPF patients were enrolled into an evaluation of the validity of the formula to predict the presence of $\mathrm{PH}$, defined as MPAP $>25 \mathrm{mmHg}$. For patients in whom the formula alerted us to the possibility of $\mathrm{PH}$, significantly lower TLCO, $\mathrm{SpO}_{2}$ and $\mathrm{PaO}_{2}$ and significantly higher $\mathrm{FVC} \% / \mathrm{DLCO} \%$ were found. No significant difference was found in terms of predicting $\mathrm{PH}$ by using the cut off points of 21 and $25 \mathrm{mmHg}$, respectively. Using the latter cut-off value, a strong correlation was found between the formula-predicted $\mathrm{PH}$ and $\mathrm{SaO}_{2}, \mathrm{PaO}_{2}$, right ventricular systolic pressure measured by echocardiography and hilar width on chest X-ray. However, the greatest limitation of this study in our opinion is the lack of a "gold standard" method verifying the PAP values. According to the authors, the accuracy of MPAP estimation was assessed by examining the correlation between the predicted MPAP using the formula and the PH diagnosed by other screening tools along with clinical signs of $\mathrm{PH}$ in the patients.

Thus, our study seems to be the only one which compares calculated data concerning the $\mathrm{PH}$ 
with values precisely measured. Certainly, the fact that the study was organised as a one centre study restricts the size of our group of IPF patients to a small number, although nonetheless comparable with the numbers in studies published so far. Also, focusing on lung transplantation candidates only lead to the decrease of included patients, However, primarily the patients with advanced disease as are the lung transplantation candidates are at highest risk of $\mathrm{PH}$ and thus they would most profit from this method. Also, right heart catheterisation used in our study to verify the $\mathrm{PH}$ is the method far for being suitable for routine use [28]. Thus, in our opinion, to perform this invasive method in IPF patients is justifiable as a part of pre-transplantation examination.

Conversely, the way the study was organised can be considered its great advantage. To our knowledge, no other study has yet been published which evaluates data concerning pulmonary hypertension assessment obtained prospectively in the same day. This organisation should eliminate the possible changes of pressures in the right ventricle and pulmonary artery in time and thus the incorrectness of obtained results. According to our experience, this method of organisation is well tolerated by the patients and they do not find it too exhausting. However, it requires great effort on the part of all the relevant healthcare professionals to co-ordinate the time schedule of the particular procedures.

In conclusion, our study did not confirm the possibility of using the formula based on lung function values to predict with sufficient accuracy either the presence or the level of $\mathrm{PH}$ in patients with IPF. Further carefully organised studies will be necessary to verify or definitively reject this formula. Till then, we must concede that no indirect method using the lung function parameters is available at present to predict with sufficient preciseness pulmonary hypertension in IPF patients. Despite its invasiveness, pulmonary catheterisation remains the "gold standard" method bringing indisputable evidence of PH. However, non-invasive examinations should be performed first to specify precisely the patients suspected of $\mathrm{PH}$. According to a recently published review, these noninvasive investigations should include echocardiography, complete lung functions tests including TLCO, measurement of BNP or NT-pro-BNP, appropriate immunological markers, overnight oximetry and CT chest angiography [29]. If these results support the presence of $\mathrm{PH}, \mathrm{RHC}$ (including vasoreactivity testing) should be performed.

\section{References}

1. American Thoracic Society/European Respiratory Society International Multidisciplinary Consensus Classification of the Idiopathic Interstitial Pneumonias. Am J Respir Crit Care Med 2002; 165: 277-304.

2. Raghu G, Weycker D, Edelsberg J, et al. Incidence and Prevalence of Idiopathic Pulmonary Fibrosis. Am J Respir Crit Care Med 2006; 174: 810-6.

3. Kolek V. Epidemiology of cryptogenic fibrosing alveolitis in Moravia and Silesia. Acta Univ Palacki Olomouc Fac Med 1994; 137: 49-50.
4. Mejía M, Carrillo G, Rojas-Serrano J, et al. Idiopathic Pulmonary Fibrosis and Emphysema. Decreased Survival Associated With Severe Pulmonary Arterial Hypertension. Chest 2009; 136: 10-5.

5. Raghu G, Collard HR, Egan JJ, et al. An official ATS/ERS/JRS/ALAT statement: Idiopathic pulmonary fibrosis: Evidence-based guidelines for diagnosis and treatment. Am J Respir Crit Care Med 2011; 183: 788-824.

6. Pérez ER, Daniels CE, Schroeder DR, et al. Incidence, prevalence, and clinical course of idiopathic pulmonary fibrosis: a population-based study. Chest 2010; 137: 129-37.

7. Daniels CE, Yi ES, Ryu JH. Autopsy findings in 42 consecutive patients with idiopathic pulmonary fibrosis. Eur Respir J 2008; 32: 170-4.

8. Nathan SD, Shlobin OA, Ahmad S, et al. Pulmonary hypertension and pulmonary function testing in idiopathic pulmonary fibrosis. Chest 2007; 131: 657-63.

9. Steen VD, Graham G, Conte C, et al. Isolated diffusing capacity reduction in systemic sclerosis. Arthritis Rheum 1992; 35: 765-70.

10. Zisman DA, Ross DJ, Belperio JA, et al. Prediction of pulmonary hypertension in idiopathic pulmonary fibrosis. Respir Med 2007; 101: 2153-9.

11. Zisman DA, Karlamangla AS, Kawut SM, et al. Validation of a method to screen for pulmonary hypertension in advanced idiopathic pulmonary fibrosis. Chest 2008; 133: 640-5.

12. Arcasoy SM, Christie JD, Ferrari VA, et al. Echocardiographic assessment of pulmonary hypertension in patients with advanced lung disease. Am J Respir Crit Care Med 2003; 167: 735-40.

13. Nathan SD, Noble PW, Tuder RM. Idiopathic pulmonary fibrosis and pulmonary hypertension. Connecting the dots. Am J Respir Crit Care Med 2007; 175: 875-80.

14. Lettieri CJ, Nathan SD, Barnett S, et al. Prevalence and outcomes of pulmonary arterial hypertension in idiopathic pulmonary fibrosis. Chest 2006; 129: 746-52.

15. King TE Jr, Tooze JA, Schwarz MI, et al. Predicting survival in idiopathic pulmonary fibrosis: scoring system and survival model. Am J Respir Crit Care Med 2001; 164: 1171-81.

16. Santos RC, Sinha A, Riar SS, et al. Utility of CT scan for detection and grading the severity of pulmonary hypertension in patients with parenchymal lung disease. Chest 2007; 132: 621S-2S.

17. Lee HJ, Babchyck B, Dedopoulos S, et al. Utilizing pulmonary artery/aortic diameter ratio from CT scans of ther chest to predict pulmonary artery hypertension. Chest 2007; 132: 624S.

18. Zisman DA, Karlamangla AS, Ross DJ, et al. High-resolution chest CT findings do not predict the presence of pulmonary hypertension in advanced idiopathic pulmonary fibrosis. Chest 2007; 132: 773-9.

19. Fisher MR, Forfia PR, Chamera E, et al. Accuracy of Doppler echocardiography in the hemodynamic assessment of pulmonary hypertension. Am J Respir Crit Care Med 2009; 179: 615-21.

20. Nathan SD, Shlobin OA, Barnett SD, et al. Right ventricular systolic pressure by echocardiography as a predictor of pulmonary hypertension in idiopathic pulmonary fibrosis. Respir Med 2008; 102: 1305-10.

21. Shorr AF, Wainright JL, Cors CS, et al. Pulmonary hypertension in patients with pulmonary fibrosis awaiting lung transplant. Eur Respir J 2007; 30: 715-21.

22. Todd NW, Lavania S, Park MH, et al. Variable prevalence of pulmonary hypertension in patients with advanced interstitial pneumonia. J Heart Lung Transplant 2010; 29: 188-94.

23. Nathan SD, Ahmad S, Shlobin OA, et al. Correlation of pulmonary function testing with pulmonary arterial hy- 
pertension $(\mathrm{PAH})$ in patients with idiopathic pulmonary fibrosis (IPF). Proc Am Thorac Soc 2006; 3: A103.

24. Nadrous HF, Pellika PA, Krowka MJ, et al. Pulmonary hypertension in patients with idiopathic pulmonary fibrosis. Chest 2005; 128: 2393-9.

25. Todd NW, Lavania S, Park MH, et al. Variable prevalence of pulmonary hypertension in patients with advanced interstitial pneumonia. J Heart Lung Transplant 2010; 29: 188-94.

26. Wells AU, Desai SR, Rubens MB, et al. Idiopathic pulmonary fibrosis. A composite physiologic index derived from disease extent observed by computed to- mography. Am J Respir Crit Care Med 2003; 167: 962-9.

27. Ghanem MK, Makhlouf HA, Agmy GR, et al. Evaluation of recently validated non-invasive formula using basic lung functions as new screening tool for pulmonary hypertension in idiopathic pulmonary fibrosis patients. Ann Thorac Med 2009; 4: 187-96.

28. Ley B, Collard HR, King, TE. Clinical course and prediction of survival in idiopathis pulmonary fibrosis. Am J Respir Crit Care Med 2011; 183: 431-40.

29. Behr J, Ryu JH. Pulmonary hypertension in interstitial lung disease. Eur Respir J 2008; 31: 1357-67.

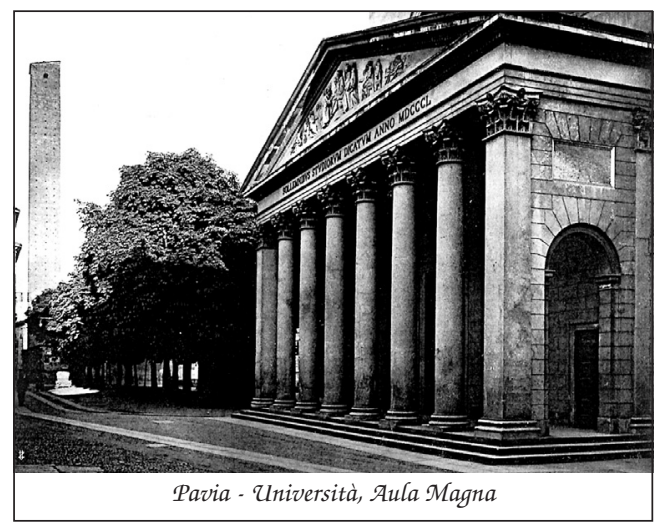

